This is the final peer-reviewed accepted manuscript of:

F. Franchi; B. Lazzari; R. Nibbi - Mathematical model for the non-isothermal Johnson-Segalman viscoelasticity in porous media: stability and wave propagation - Mathematical methods in the Applied Sciences, vol. 38 (2015), pp. 4075-4087.

The final published version is available online at: https://doi.org/10.1002/mma.3348

Rights / License:

The terms and conditions for the reuse of this version of the manuscript are specified in the publishing policy. For all terms of use and more information see the publisher's website.

This item was downloaded from IRIS Università di Bologna (https://cris.unibo.it/)

When citing, please refer to the published version. 


\title{
Mathematical models for the non-isothermal Johnson-Segalman viscoelasticity in porous media: stability and wave propagation
}

\author{
Franca Franca, Barbara Lazzari*and Roberta Nibbi
}

We present a nonlinear model for J-S type polymeric fluids in porous media, accounting for thermal effects of Oldroyd-B type. We provide a thermodynamic development of a Darcy theory which is consistent with the interlacement between thermal and viscoelastic relaxation effects and diffusion phenomena. The appropriate invariant convected time derivative for the flux vector and the stress tensor is discussed.

This is performed by investigating the local balance laws and entropy inequality in the spatial configuration, within the single fluid approach. For constant parameters, our thermomechanical setting is of Jeffreys-type with two delay time parameters, and hence in the linear/linearized version it is strictly related to phase-lag theories within first-order Taylor approximations. A detailed spectral analysis is carried out for the linearized version of the model, with a scrutiny to some significant limit situations, enhancing the stabilizing effects of the dissipative and elastic mechanisms, also for retardation responses.

For polymeric liquids, rheological aspects, wave propagation properties and analogies with other theories with lagging are pointed out.

Copyright (c) 0000 John Wiley \& Sons, Ltd.

Keywords: Porous media; Viscoelastic fluids; Non-Fourier heat conduction; Thermodynamics; Phase-lag;

Stability; Hyperbolic waves

\section{Introduction}

Non-Newtonian materials with fading memory (polymers, fibre suspensions, blood ...) exhibit a behavior that is intermediate between the nonlinear hyperbolic response of purely elastic materials and the likewise strong diffusion parabolic response of viscous fluids. Under shear, complex fluids often undergo instabilities leading to new flow patterns which are triggered by the nonlinear terms of the invariant rate-type equation for the elastic stress tensor. Their non-ideal, hysteretic and confinement properties become challenging aspects to be taken into account in view of mathematical modellings.

The general Johnson-Segalman (J-S) model seems to provide a useful description for diluted suspensions of a high molecular weight polymer into a largely viscous solvent, which may be generally considered as a liquid. As a special case also purely polymeric solutions are properly accounted for. In this way, rather than the solvent-solution mixture approach, the single-fluid frame is addressed. Here, we restrict our attention to the corotational J-S model, whose non-instantaneous response character is embodied in a rate-type constitutive equation for the elastic polymeric stress tensor. This model is very flexible and furnishes an interesting mathematical formulation that is easily incorporated also into the boundary layer theories for engineering applications. It is a viscoelastic model of Oldroyd-B type and includes, as special sub-classes, the classical Newtonian and Maxwell models.

On the other hand, in many physical, astrophysical and biomedical situations, the influence of the temperature is important and, hence, it is unlike to be negligible. So we, in a close analogy with the J-S type constitutive structure, we propose also a non-Fourier heat transfer theory.

Department of Mathematics, University of Bologna, Piazza di Porta San Donato 5, 40126 Bologna Italy

* Correspondence to: B. Lazzari, Department of Mathematics, University of Bologna, Piazza di Porta San Donato 5, 40126 Bologna Italy email: barbara.lazzari@unibo.it 
Besides, several applications (see e.g. [1]) require to account for the presence of porous effects. It is well known that the goodness of a mathematical model describing the porous media behavior is strictly related to the porosity (ranging from zero to one) of the medium under consideration. Thinking of future applications in biomedical and astrophysical settings, in this paper we focus our attention to non-isothermal viscoelastic flows in porous media well described by Darcy's law. Indeed, the Darcy model can adequately capture the effects of porous media in many practical situations when the porosity is not high (that is to say, it is not close to one).

On the other hand, it is often argued that, for porosities close to one as happens in metallic foams, the Brinkman model, including an additional viscosity term, turn out to be more appropriate (see e.g. [2]). To this purpose, we believe that our model may be easily generalized to account for also high porosity materials, since an analogous viscosity term is already present within the J-S viscoelasticity.

So, in this paper, within an extended thermodynamic scheme and for a special choice of the Helmholtz free energy, we deliver a nonlinear thermo-viscoelastic J-S model for dilute polymer solutions in a Darcy porous context, where the coupled mechanical and thermal effects exhibit similar rheological properties, accounting for the necessary dissipation mechanisms and non-instantaneous responses, through the elastic components.

For constant parameters, our constitutive setting is of Jeffreys type with a relaxation and a retardation time. Only within the linear/linearized frame, and for small delay parameters, we point out the special link with other phase-lag theories by Tzou [3]; in the same context, the relation between the J-S and the "backwards" Kelvin-Voight models [4] is also stressed. As a special case, we confine our attention on the destabilizing advance behavior of Walters-B type and the stabilizing retardation response of Rivlin-Ericksen type.

In view of the previous remarks, our model seems to better describe the behavior of complex fluids; moreover, it is always possible to generalize it in order to account for cellular and masses aggregation formation processes.

Interestingly, for human endothelial cells, several experiments show the formation of complex structures like clusters and network patterns, both filaments and sprouts, that can be interpreted as the beginning of a vasculature: this phenomenon is responsible of the tumor-induced angiogenesis. These structures cannot be explained by parabolic models, but are recovered through numerical simulations on hyperbolic models [5]. Hyperbolic systems are so employed to study the intermediate regime at the macroscopic level, for the cell density, whereas the parabolic flux gradient context for the chemoattractant is always preserved (see, for example, [6, 7]).

By introducing a chemotactic force proportional to the chemoattractant gradient in the momentum equation, our hydrodynamic theory may also describe a chemotactic aggregation process, in presence of delay parameters, thus generalyzing the model proposed in [5]. Obviously, we need an enlargement of the governing system to include an evolution equation for the chemoattractant concentration. One possibility is to consider the same parabolic reaction-diffusion equation as in the pioneering Keller-Segel model [8], but, even if an interacting hyperbolic-parabolic system seems to be still legitimated both mathematically and experimentally [9], in our opinion a totally hyperbolic system should give rise to a novel challenging research issue.

In order to interrogate our model, we also perform a judicious Fourier Modes tool for developing a linear stability analysis and comment the general dispersion equation for transverse and longitudinal modes. Thanks to the joint dissipative and elastic properties we always recover asymptotic stability, also in the presence of retardation effects. These stability results assess the goodness of the model in view of its future applications.

For some purposes it has been proved useful to impose the constraint of incompressibility on the flow velocity, which is also a commonly legitimated approximation in solar and astrophysical settings [10]; in our viscoelastic context, this leads to some rheological analogies. In particular, for purely polymeric melts in a Darcy porous medium, it could be proved the existence of nonlinear transverse shear waves, exhibiting a torsional character, related to the corotational convected time derivative. Meanwhile, these viscoelastic waves could share analogies with the existence of incompressible torsional Alfvén waves in magnetic flux tubes. Since their recent detection [11], these magnetic waves are thought to play a crucial role in solar coronal heating events, in magneto-seismology and in the formation of complex structures, like clusters and network patterns (filaments, sprouts and spicule-like features), that is the so called "cosmic web" [12, 13, 14, 15, 16, 17]. It is generally accepted that the framework for the formation of such outstanding structural elements is just that of gravitational instability. By comparison, we conjecture that also the incompressible poroviscoelastic shear waves might manifest a similar experimental importance in studies concerning the formation of complex structures within chemotactic and gravitational collapses $[5,18]$.

The paper is organized as follows. In Section 2 we furnish some preliminaries and the thermodynamical scheme; thermodynamic developments are presented in Section 3, whereas in Section 4, from a special choice of the free energy potential, we derive the general governing system. Subsequently, in Section 5, a spectral tool is performed to investigate the linear stability problem; finally, some comments on possible applications and generalizations of our model close the paper.

\section{Preliminaries and thermodynamic scheme}

Throughout the paper we consider a $3-D$ body $\mathcal{B}$ and denote by $\mathcal{R}_{t}$ in $\mathbb{R}^{3}$ the smooth region occupied by $\mathcal{B}$ in the present configuration. For non-isothermal flows, besides the constitutive setting, it is necessary to include the energy equation in order to enhance, among various terms, viscous dissipation aspects: it is known that many non-Newtonian fluids are highly viscous, and hence, they exhibit a non negligible viscous dissipation. This consideration suggests to use a non-isothermal approach, where 
thermal and mechanical phenomena can be considered coupled.

For our present purposes, we need to provide a heat conduction theory for a J-S type viscoelastic fluid, flowing in a porous material, modelling a solvent/solution polymeric fluid. Consequently, we work with spatial configurations, even if we omit all spatial dependences; so we refer to the classical formulation of the local balance equations within the one-fluid approach.

In a non-isothermal polymeric solution, described through a viscoelasticity of J-S type, the total symmetric viscous stress tensor $\boldsymbol{\Pi}$ is given by the sum of a Newtonian/solvent part $\boldsymbol{\Pi}_{s}$ and an elastic part $\boldsymbol{\Pi}_{e}$, due to the presence of the polymeric solutions, which satisfy the following (stationary and evolution) constitutive equations:

$$
\begin{aligned}
& \boldsymbol{\Pi}_{s}=2 \mu_{s} \mathbf{D}+\left(\zeta_{s}-\frac{2 \mu_{s}}{3}\right)(\operatorname{tr} \mathbf{D}) \mathbb{I}, \\
& \tau_{\pi_{e}}\left(\frac{D \boldsymbol{\Pi}_{e}}{D t}\right)_{J}+\boldsymbol{\Pi}_{e}=2 \mu_{e} \mathbf{D}+\left(\zeta_{e}-\frac{2 \mu_{e}}{3}\right)(\operatorname{tr} \mathbf{D}) \mathbb{I},
\end{aligned}
$$

where $\mathbf{D}$ is the symmetric part of the velocity gradient $\mathbf{L}=\nabla \mathbf{v}, \mu_{s}$ and $\mu_{e}$ are the solvent and elastic shear viscosities, while $\zeta_{s}$ and $\zeta_{e}$ are the solvent and elastic bulk viscosities.

The notation $\left(\frac{D}{D t}\right)$, stands for the corotational or Jaumann derivative, defined as follows

$$
\left(\frac{D \boldsymbol{\Pi}_{e}}{D t}\right)_{\jmath}=\dot{\boldsymbol{\Pi}}_{e}+\boldsymbol{\Pi}_{e} \mathbf{W}-\mathbf{W} \boldsymbol{\Pi}_{e}
$$

where the superposed dot denotes the material time derivative, while $\mathbf{W}$ is the skew-symmetric part of $\mathbf{L}$.

In most papers the viscosity parameters and the relaxation time $\tau_{\pi_{e}}$ are supposed to be constant and positive. It is experimentally shown that $\mu_{s}>\mu_{e}$ and also $\mu>\mu_{e}, \mu$ being the total shear viscosity and the analogous holds for the bulk viscosities.

As it is known, the nonlinear invariant $\mathrm{J}$-S model provides a non-instaneous response character, which is embodied in the constitutive equation of rate-type for the elastic stress tensor, with a single relaxation time and elastic viscosities (see [19]). In particular, it seems legitimated to regard a blood capillary as a viscoelastic material, where elastic and viscous properties are joined together, as experimentally confirmed for examples in flux tubes [20]. Meanwhile this model can also reproduce angiogenesis experiments under a variety of physiological conditions. Examples of blow-up are captured by numerical approximations and global solutions are recovered by means of slow growth processes, which in turn validate also the linear stability analysis [21].

In order to provide a non-Fourier heat theory for a non-isothermal polymeric solution of J-S type, accounting for also delayed thermal effects, we address our attention to a non-instantaneous thermal response, which may be based on an analogous constitutive setting. So we propose a split form for the entropy flux vector $\mathbf{q} / \Theta, \Theta>0$ being the absolute temperature, as follows:

$$
\frac{\mathbf{q}}{\Theta}=\frac{\mathbf{q}_{F}}{\Theta}+\frac{\mathbf{q}_{e}}{\Theta} .
$$

The Fourier/diffusion flux vector $\mathbf{q}_{F}$ and the elastic flux vector $\mathbf{q}_{e}$ satisfy the following constitutive equations of stationary and rate-type, respectively:

$$
\begin{aligned}
\mathbf{q}_{F} & =-k_{F} \nabla \Theta, \\
\tau_{\mathrm{qe}}\left(\frac{D \mathbf{q}_{e}}{D t}\right)_{J}+\mathbf{q}_{e} & =-k_{e} \nabla \Theta,
\end{aligned}
$$

where the Fourier and elastic thermal diffusivity parameters $k_{F}, k_{e}$ and the thermal relaxation time $\tau_{\mathrm{q}_{e}}$ are generally viewed as positive and constant. In this case $\left(\frac{D}{D t}\right)$, assumes the form

$$
\left(\frac{D \mathbf{q}_{e}}{D t}\right)_{J}=\dot{\mathbf{q}}_{e}-\mathbf{W} \mathbf{q}_{e}
$$

Remark 1 It is worth to note that, only for constant parameters, the J-S viscoelastic model is of Oldroyd-B type, with the additional advantage of being described through a constitutive equation of Jeffreys-type with a relaxation time $\tau_{\pi}=\tau_{\pi_{e}}$ and a retardation time $\tau_{D}$, as follows

$$
\tau_{\pi}\left(\frac{D \boldsymbol{\Pi}}{D t}\right)_{\jmath}+\boldsymbol{\Pi}=2 \mu\left[\mathbf{D}+\tau_{D}\left(\frac{D \mathbf{D}}{D t}\right)_{J}\right]+\frac{3 \zeta-2 \mu}{3}\left[\nabla \cdot \mathbf{v}+\tau_{D}\left(\frac{D(\nabla \cdot \mathbf{v})}{D t}\right)_{J}\right] \mathbb{I} .
$$

The classical (parabolic) Newtonian model and the (hyperbolic) Maxwell type viscoelasticity are recovered for the boundary values of the dimensionless retardation parameter $r_{\pi}=\tau_{D} / \tau_{\pi}\left(0<r_{\pi}<1\right)$.

Moreover, this versatile model, in its linear/linearized version, is strictly related to the single/dual-phase-lag heat theories by Tzou [3], when combined with first order Taylor series approximations, accounting for short memory elastic effects, which happens whenever the delay parameters are suitable small (see also [22] and references therein).

Indeed the same rheological comments hold for the proposed thermal constitutive setting (see [23]). 
Remark 2 Other objective time derivatives for tensorial and vectorial fluxes are employed in the literature (see e.g. [24, 25]). Among them, we recall the (lower convected) Oldroyd type derivative for $\boldsymbol{\Pi}_{e}$ and also the analogous one for $\mathbf{q}_{e}$, defined, respectively, as follows

$$
\dot{\boldsymbol{\Pi}}_{e}-\mathbf{L} \boldsymbol{\Pi}_{e}-\boldsymbol{\Pi}_{e} \mathbf{L}^{T}+\nabla \cdot \mathbf{v} \boldsymbol{\Pi}_{e}, \quad \dot{\mathbf{q}}_{e}-\mathbf{L} \mathbf{q}_{e}+\mathbf{q}_{e} \nabla \cdot \mathbf{v},
$$

where an invariant compressional character is just expressed by the additional divergence term.

In most recent papers, the objective time derivative for the heat flux vector is labeled as the Christov convected derivative (see e.g. [26]) and the relative evolution equation $(2.1)_{2}$ is known as the Cattaneo-Christov constitutive equation (see e.g. $[27])$.

\section{Thermodynamic developments: restrictions on the constitutive responses and evolution equations}

In this section, we provide a typical extended thermodynamic development of the classical Darcy law theory, which is consistent with a constitutive behavior of J-S type for thermo-mechanical effects, and accounts for non-constant parameters too.

In some limit situations, our general thermodynamic theory governs a hyperbolic model and hence allows for the propagation of thermo-viscoelastic-poroacoustic nonlinear waves.

We begin by recapitulating the local field equations in their standard one fluid form, a few notational changes apart.

Since we are mainly interested in modelling the non-isothermal flow of non-Newtonian fluids in a Darcy type porous medium, we reformulate the momentum equation in terms of the intrinsic fluid velocity $\mathbf{v}$, rather than the usual seepage/Darcy velocity $\mathbf{v}_{D}$ (see e.g. [28] and references therein). The major advantage of this choice is that the momentum equation quickly generalizes in a natural way to incorporate other terms, such as viscosity terms, or uniform rotations expressed by a Coriolis force.

Following the more general classical formulation as in [29], our theory is based on the local balance equations for mass, momentum and energy, in the form

$$
\begin{aligned}
& \dot{\rho}+\rho \nabla \cdot \mathbf{v}=0, \\
& \rho \dot{\mathbf{v}}=-\nabla p+\rho \mathbf{b}-\mu_{D} R \mathbf{v}+\nabla \cdot \boldsymbol{\Pi}, \\
& \rho \dot{e}=\mathcal{P}_{m}^{i}+\mathcal{P}_{h}^{i},
\end{aligned}
$$

where, as already mentioned, the superposed dot denotes the material time derivative. The quantities $\rho, \mathbf{v}, p, \mathbf{b}, \boldsymbol{\Pi}$ and $e$ are, respectively, the density, velocity, pressure, external body force, total (viscous) stress tensor and internal energy, while $\mu_{D}>0$ is the so called dynamic viscosity.

Finally, in writing the momentum equation, taking into account the Dupuit-Forchheimer relation and following the Nield's strategy, we have introduced the "retardability" $R$ defined as

$$
R=\frac{\varepsilon}{k_{p}}>0
$$

where $k_{p}$ is the standard permeability and $\varepsilon(0<\varepsilon<1)$ represents the porosity of the medium.

Here, as in [19], we decompose the symmetric solvent and elastic stress tensors into the spherical and deviatoric parts, with

$$
p_{s}=-\frac{1}{3} \operatorname{tr} \Pi_{s}, \quad p_{e}=-\frac{1}{3} \operatorname{tr} \Pi_{e}
$$

and

$$
\left\langle\boldsymbol{\Pi}_{s}\right\rangle=\boldsymbol{\Pi}_{s}+p_{s} \mathbb{I}, \quad\left\langle\boldsymbol{\Pi}_{e}\right\rangle=\boldsymbol{\Pi}_{e}+p_{e} \mathbb{I}, \quad\langle\mathbf{D}\rangle=\mathbf{D}-\frac{1}{3} \nabla \cdot \mathbf{v} \mathbb{I} .
$$

In order to incorporate both the split forms for the heat flux vector and for the viscous stress tensor, the internal mechanical power density $\mathcal{P}_{m}^{i}$ and the internal heat power density $\mathcal{P}_{h}^{i}$ are here given by ${ }^{\dagger}$

$$
\begin{aligned}
& \mathcal{P}_{m}^{i}=\boldsymbol{\Pi} \cdot \mathbf{D}-p \nabla \cdot \mathbf{v}=\left(\left\langle\boldsymbol{\Pi}_{s}\right\rangle+\left\langle\boldsymbol{\Pi}_{e}\right\rangle\right) \cdot\langle\mathbf{D}\rangle-\left(p+p_{s}+p_{e}\right) \nabla \cdot \mathbf{v}, \\
& \mathcal{P}_{h}^{i}=\rho h
\end{aligned}
$$

where $h$ is the rate at which the heat is absorbed per unit mass and satisfies the heat balance equation [30]

$$
\rho h=-\nabla \cdot\left(\mathbf{q}_{F}+\mathbf{q}_{e}\right)+\rho r
$$

${ }^{\dagger}$ Clearly, both $\mathcal{P}_{m}^{i}$ and $\mathcal{P}_{h}^{i}$ will assume different expressions, depending on the non-local properties of the material under study (see e.g. [29]). In particular, non-local capillary properties of polymeric fluids, fitting for blood capillary growth, may be properly captured by an additional capillary stress tensor contribution of Korteweg type (see e.g. [21]) 
$r$ being the heat supply.

The local form of the entropy inequality is taken as follows

$$
\rho \dot{\eta} \geq \rho \frac{h}{\Theta}+\frac{1}{\Theta} \nabla \Theta \cdot\left(\frac{\mathbf{q}_{F}}{\Theta}+\frac{\mathbf{q}_{e}}{\Theta}\right)
$$

where $\eta$ is the entropy density.

It is worth to observe that multiplying (3.2) by the coldness $\Theta^{-1}$, we obtain the identity

$$
\rho \frac{h}{\Theta}=-\frac{1}{\Theta} \nabla \cdot \frac{\mathbf{q}_{F}+\mathbf{q}_{e}}{\Theta}+\frac{1}{\Theta} \rho r=-\nabla \cdot\left(\frac{\mathbf{q}_{F}+\mathbf{q}_{e}}{\Theta}\right)+\frac{1}{\Theta} \rho r-\frac{1}{\Theta} \nabla \Theta \cdot \frac{\mathbf{q}_{F}+\mathbf{q}_{e}}{\Theta},
$$

from which the internal and external entropy powers can be defined as

$$
\begin{gathered}
\mathcal{P}_{\eta}^{i}=\rho \frac{h}{\Theta}+\frac{1}{\Theta} \nabla \Theta \cdot \frac{\mathbf{q}_{F}+\mathbf{q}_{e}}{\Theta}, \\
\mathcal{P}_{\eta}^{e}=-\nabla \cdot\left(\frac{\mathbf{q}_{F}+\mathbf{q}_{e}}{\Theta}\right)+\frac{1}{\Theta} \rho r .
\end{gathered}
$$

We observe that the usual proportionality relation between the energy and entropy inflows is still preserved.

In compliance with the Second Law of Thermodynamics, we assume that (3.3) holds for all admissible processes. and, replacing $\rho h \Theta^{-1}$ by means of the energy equation, it becomes

$$
\rho \Theta \dot{\eta} \geq \rho \dot{e}-\mathcal{P}_{m}^{i}+\nabla \Theta \cdot \frac{\mathbf{q}_{F}+\mathbf{q}_{e}}{\Theta}
$$

Hence, in terms of the Helmholtz free energy $\psi=e-\Theta \eta$, (3.4) can be rewritten in the Clausius-Duhem form

$$
\rho(\dot{\Psi}+\dot{\Theta} \eta)-\left(\left\langle\boldsymbol{\Pi}_{s}\right\rangle+\left\langle\boldsymbol{\Pi}_{e}\right\rangle\right) \cdot\langle\mathbf{D}\rangle+\left(p+p_{s}+p_{e}\right) \nabla \cdot \mathbf{v}+\nabla \Theta \cdot \frac{\mathbf{q}_{F}+\mathbf{q}_{e}}{\Theta} \leq 0 .
$$

In order to provide our constitutive theory, within the (non-equilibrium) extended thermodynamics framework, we let $\Gamma=$ $\left(\rho, \Theta, \nabla \Theta, \mathbf{D}, \mathbf{q}_{e},\left\langle\boldsymbol{\Pi}_{e}\right\rangle, p_{e}\right)$ be the enlarged set of the independent state variables. Then we assume $\Psi, e, \eta$ and $p$ be continuously differentiable functions of $\Gamma$. Making use of the chain rule, inequality (3.5), easily gives

$$
\begin{array}{r}
\rho\left(\Psi_{\Theta}+\eta\right) \dot{\Theta}-\left(\rho \Psi_{\rho}-\frac{p}{\rho}\right) \dot{\rho}+\rho \Psi_{\nabla \Theta} \cdot \dot{\nabla} \dot{\nabla}+\rho \Psi_{\mathrm{D}} \cdot \dot{\mathbf{D}}+\rho \Psi_{\mathrm{q}_{e}} \cdot \dot{\mathbf{q}}_{e}+\rho \Psi_{\left\langle\Pi_{e}\right\rangle} \cdot \overline{\left\langle\boldsymbol{\Pi}_{e}\right\rangle}+\rho \Psi_{p_{e}} \dot{p}_{e}-\left(\left\langle\boldsymbol{\Pi}_{s}\right\rangle+\left\langle\boldsymbol{\Pi}_{e}\right\rangle\right) \cdot\langle\mathbf{D}\rangle \\
+\left(p_{s}+p_{e}\right) \nabla \cdot \mathbf{v}+\nabla \Theta \cdot \frac{\mathbf{q}_{F}+\mathbf{q}_{e}}{\Theta} \leq 0
\end{array}
$$

where the subscripts stand for the partial derivatives.

In consistence with the fact that phenomenological statements must be linear in the (extra) variables, vanishing in equilibrium, we henceforth suppose that

$$
\rho \Psi_{\mathrm{q}_{e}}=\hat{\alpha}_{e} \mathbf{q}_{e}, \quad \rho \Psi_{\left\langle\Pi_{e}\right\rangle}=\beta_{e}\left\langle\boldsymbol{\Pi}_{e}\right\rangle, \quad \rho \Psi_{p_{e}}=\gamma_{e} p_{e},
$$

where the parameters $\hat{\alpha}_{e}, \beta_{e}$ and $\gamma_{e}$ may depend at most on $(\mathbf{x}, t), \rho$ and $\Theta$. It is worth to stress that the positiveness of such scalar valued functions is strictly related to stability requirements. In a simplified context $\hat{\alpha}_{e}, \beta_{e}$ and $\gamma_{e}$ may be regarded as constant.

The substitution of (3.7) in (3.6), together with a troublesome rearrangement of some terms, yields ${ }^{\ddagger}$

$$
\begin{array}{r}
\left(\rho \Psi_{\rho}-\frac{p}{\rho}\right) \dot{\rho}-\rho\left(\Psi_{\Theta}+\eta\right) \dot{\Theta}-\rho \Psi_{\nabla \Theta} \cdot \dot{\nabla \Theta}-\rho \Psi_{\mathrm{D}} \cdot \dot{\mathbf{D}}+\left\langle\boldsymbol{\Pi}_{s}\right\rangle \cdot\langle\mathbf{D}\rangle-p_{s} \nabla \cdot \mathbf{v}-\frac{\mathbf{q}_{F}}{\Theta} \cdot \nabla \Theta+\left\langle\boldsymbol{\Pi}_{e}\right\rangle \cdot\left(\langle\mathbf{D}\rangle-\beta_{e} \overline{\left\langle\boldsymbol{\Pi}_{e}\right\rangle}\right) \\
-p_{e}\left(\gamma_{e} \dot{p}_{e}+\nabla \cdot \mathbf{v}\right)-\frac{\mathbf{q}_{e}}{\Theta} \cdot\left(\hat{\alpha}_{e} \Theta \dot{q}_{e}+\nabla \Theta\right) \geq 0 .
\end{array}
$$

$\ddagger$ We also note that, in view of (3.7), the material time derivative of the deviatoric elastic stress tensor and of the elastic heat flux vector may be replaced by the objective corotational/Jaumann convected time derivative, namely

$$
\begin{gathered}
\left\langle\Pi_{e}\right\rangle \cdot \overline{\left\langle\Pi_{e}\right\rangle}=\left\langle\Pi_{e}\right\rangle \cdot\left(\frac{\dot{\left\langle\Pi_{e}\right\rangle}}{-W} \Pi_{e}+\Pi_{e} \mathrm{~W}\right)=\left\langle\Pi_{e}\right\rangle \cdot\left(\frac{D\left\langle\Pi_{e}\right\rangle}{D t}\right)_{J}, \\
\mathrm{q}_{e} \cdot \dot{\mathrm{q}}_{e}=\mathrm{q}_{e} \cdot\left(\dot{\mathrm{q}}_{e}-\mathrm{Wq} \mathrm{q}_{e}\right)=\mathrm{q}_{e} \cdot\left(\frac{D \mathrm{q}_{e}}{D t}\right)_{J} .
\end{gathered}
$$


The arbitrariness of $\dot{\rho}, \dot{\Theta}, \dot{\nabla} \Theta$ and $\mathbf{D}$ implies that

$$
p=\rho^{2} \Psi_{\rho}, \quad \eta=-\Psi_{\ominus}, \quad \Psi_{\nabla \Theta}=0, \quad \Psi_{\mathrm{D}}=0,
$$

that is to say that $\Psi$ can only depend on $\rho, \Theta,\left\langle\boldsymbol{\Pi}_{e}\right\rangle, p_{e}$ and $\mathbf{q}_{e}$.

From the split residual entropy inequality we recover the classical Navier-Stokes/Fourier restrictions

$$
\left\langle\boldsymbol{\Pi}_{s}\right\rangle \cdot\langle\mathbf{D}\rangle \geq 0, \quad-p_{s} \nabla \cdot \mathbf{v} \geq 0, \quad-\frac{\mathbf{q}_{F}}{\Theta} \cdot \nabla \Theta \geq 0
$$

and the following further restrictions for the elastic terms

$$
\left\langle\boldsymbol{\Pi}_{e}\right\rangle \cdot\left(\langle\mathbf{D}\rangle-\beta_{e} \overline{\left\langle\dot{\boldsymbol{\Pi}}_{e}\right\rangle}\right) \geq 0,-p_{e}\left(\gamma_{e} \dot{p}_{e}+\nabla \cdot \mathbf{v}\right) \geq 0,-\frac{\mathbf{q}_{e}}{\Theta} \cdot\left(\hat{\alpha}_{e} \Theta \dot{\mathbf{q}}_{e}+\nabla \Theta\right) \geq 0 .
$$

Inequalities (3.9) hold if

$$
\left\langle\boldsymbol{\Pi}_{s}\right\rangle=2 \mu_{s}\langle\mathbf{D}\rangle, \quad p_{s}=-\zeta_{s} \nabla \cdot \mathbf{v}, \quad \frac{\mathbf{q}_{F}}{\Theta}=-\hat{k}_{F} \nabla \Theta,
$$

where $\mu_{s}, \zeta_{s}$ and $\hat{k}_{F}$ are positive valued functions, depending on at most $(\mathbf{x}, t), \rho$ and $\Theta ; \mu_{s}, \zeta_{s}$ play the roles of solvent shear and bulk viscosities, while $k_{F}=\hat{k}_{F} \Theta$ stands for the Fourier thermal conductivity.

Finally, inequalities (3.10), in turn, hold if we choose

$$
\begin{aligned}
\left\langle\boldsymbol{\Pi}_{e}\right\rangle & =-2 \mu_{e}\left(\beta_{e} \overline{\left\langle\boldsymbol{\Pi}_{e}\right\rangle}-\langle\mathbf{D}\rangle\right) \\
p_{e} & =-\zeta_{e}\left(\gamma_{e} \dot{p}_{e}+\nabla \cdot \mathbf{v}\right) \\
\frac{\mathbf{q}_{e}}{\Theta} & =-\hat{k}_{e}\left(\hat{\alpha}_{e} \Theta \dot{\mathbf{q}}_{e}+\nabla \Theta\right)
\end{aligned}
$$

$\mu_{e}, \zeta_{e}$ and $\hat{k}_{e}$ being positive valued functions, depending on at most $(\mathbf{x}, t), \rho$ and $\Theta$. The functions $\mu_{e}$, $\zeta_{e}$ may be interpreted as the elastic shear and bulk viscosities, whereas $k_{e}=\hat{k}_{e} \Theta$ as the elastic conductivity.

Taking into account that the two first equations represent respectively the deviatoric and spherical part of the same rate-type constitutive equation for the elastic stress tensor $\boldsymbol{\Pi}_{e}$, we need to have $\gamma_{e}=2 \frac{\mu_{e}}{\zeta_{e}} \beta_{e}$.

Moreover, letting $\alpha_{e}=\hat{\alpha}_{e} \Theta$, if we define the (visco)elastic relaxation time as $\tau_{\pi_{e}}=2 \beta_{e} \mu_{e}$ and the thermal relaxation time as $\tau_{q_{e}}=\alpha_{e} k_{e}$, from (3.11) we derive the evolution equations in the usual rate-type form for $\left\langle\boldsymbol{\Pi}_{e}\right\rangle, p_{e}$ and $\mathbf{q}_{e}$ :

$$
\begin{aligned}
\tau_{\pi_{e}} \overline{\left\langle\boldsymbol{\Pi}_{e}\right\rangle}+\left\langle\boldsymbol{\Pi}_{e}\right\rangle & =2 \mu_{e}\langle\mathbf{D}\rangle, \\
\tau_{\pi_{e}} \dot{p}_{e}+p_{e} & =-\zeta_{e} \nabla \cdot \mathbf{v}, \\
\tau_{q_{e}} \dot{\mathbf{q}}_{e}+\mathbf{q}_{e} & =-k_{e} \nabla \Theta .
\end{aligned}
$$

Remark 3 Interestingly the rate-type equation for $\mathbf{q}_{e}$ may be easily rewritten as an evolution equation for the elastic entropy flux vector $\frac{q_{e}}{\Theta}$ of the form

$$
\tau_{q_{e}} \frac{\mathrm{d}}{\mathrm{dt}}\left(\frac{\mathbf{q}_{e}}{\Theta}\right)+\frac{\mathbf{q}_{e}}{\Theta}=-\hat{k}_{e} \nabla \Theta-\alpha_{e} \hat{k}_{e} \frac{\mathbf{q}_{e}}{\Theta} \dot{\Theta}
$$

This equation for $\frac{q_{e}}{\Theta}$ is compatible with the Second Law of Thermodynamics and is a generalization of the Cattaneo-type equation for $\mathbf{q}_{e}$; moreover it may be compared with the constitutive equation recovered in [31], within the different framework of internal variables.

Indeed whenewer $\tau_{q_{e}}=0$, that is to say when $\alpha_{e}=0$, the total entropy flux vector is given by the Fourier form

$$
\frac{\mathbf{q}}{\Theta}=-\left(\hat{k}_{F}+\hat{k}_{e}\right) \nabla \Theta=-\hat{k} \nabla \Theta
$$

which in turn, within the rigid heat conduction, allows for recovering a nonlinear degenerate parabolic equation, known in literature as a PME with exponent 2. It is worth to remember that such equation exhibits the remarkable mathematical property of finite wavespeed propagation (see e. g. [32]).

However, the classical linear parabolic heat equation follows for $\hat{k}=k_{0} / \Theta, k_{0}$ being a positive constant playing the role of thermal mobility.

\section{A thermodynamic model for the J-S type viscoelasticity accounting for thermal effects of Oldroyd-B type in a porous medium}

In the light of the results of the previous section, we confine our attention to free energy potentials of the following quadratic type

$$
\Psi\left(\rho, \Theta,\left\langle\boldsymbol{\Pi}_{e}\right\rangle, p_{e}, \mathbf{q}_{e}\right)=\bar{\Psi}(\rho, \Theta)+\frac{\tau_{q}}{2 \rho k_{e} \Theta}\left|\mathbf{q}_{e}\right|^{2}+\frac{\tau_{\pi}}{2 \rho}\left(\frac{\left|\left\langle\boldsymbol{\Pi}_{e}\right\rangle\right|^{2}}{2 \mu_{e}}+\frac{p_{e}^{2}}{\zeta_{e}}\right)
$$


where, for simplicity in writing, we let $\tau_{q_{e}}=\tau_{q}$ and $\tau_{\Pi_{e}}=\tau_{\Pi}$

By employing the definition of $\Psi$ and (3.8), we easily get

$$
\begin{aligned}
& p=\rho^{2} \Psi_{\rho}=\rho^{2} \bar{\Psi}_{\rho}+\frac{1}{2}\left(\rho\left(\frac{\tau_{q}}{k_{e} \Theta}\right)_{\rho}-\frac{\tau_{q}}{k_{e} \Theta}\right)\left|\mathbf{q}_{e}\right|^{2}+\frac{1}{2}\left(\rho\left(\frac{\tau_{\pi}}{2 \mu_{e}}\right)_{\rho}-\frac{\tau_{\pi}}{2 \mu_{e}}\right)\left|\left\langle\boldsymbol{\Pi}_{e}\right\rangle\right|^{2}+\frac{1}{2}\left(\rho\left(\frac{\tau_{\pi}}{\zeta_{e}}\right)_{\rho}-\frac{\tau_{\pi}}{\zeta_{e}}\right) p_{e}^{2}, \\
& \eta=-\Psi_{\Theta}=-\bar{\Psi}_{\Theta}-\frac{1}{2}\left(\frac{\tau_{q}}{\rho k_{e} \Theta}\right)_{\Theta}\left|\mathbf{q}_{e}\right|^{2}-\frac{1}{2}\left(\frac{\tau_{\pi}}{2 \rho \mu_{e}}\right)_{\Theta}\left|\left\langle\boldsymbol{\Pi}_{e}\right\rangle\right|^{2}+\frac{1}{2}\left(\frac{\tau_{\pi}}{\rho \zeta_{e}}\right)_{\Theta} p_{e}^{2} \\
& \rho e=\rho\left(\bar{\Psi}-\Theta \bar{\Psi}_{\Theta}\right)+\frac{1}{2}\left(\frac{\tau_{q}}{k_{e} \Theta}-\left(\frac{\tau_{q}}{k_{e}} \Theta\right)_{\Theta} \Theta\right)\left|\mathbf{q}_{e}\right|^{2}+\frac{1}{2}\left(\frac{\tau_{\pi}}{2 \mu_{e}}-\left(\frac{\tau_{\pi}}{2 \mu_{e}}\right)_{\Theta} \Theta\right)\left|\left\langle\boldsymbol{\Pi}_{e}\right\rangle\right|^{2}+\frac{1}{2}\left(\frac{\tau_{\pi}}{\zeta_{e}}-\left(\frac{\tau_{\pi}}{\zeta_{e}}\right)_{\Theta} \Theta\right) p_{e}^{2} .
\end{aligned}
$$

It is worth to observe that $p$ and $e$ depend on the extra variables also when the ratios $\frac{\tau_{q}}{k_{e} \Theta}, \frac{\tau_{\pi}}{2 \mu_{e}}$ and $\frac{\tau_{\pi}}{\zeta_{e}}$ are taken constant. On the contrary, the classical case $e=e(\rho, \Theta)$ may be readily recovered whenever the same ratios exhibit a homogeneous linear dependence on $\Theta$.

In the general case, as a consequences of relations (3.1) 1 and (3.8), the time derivative of the internal energy assumes the form

$$
\begin{aligned}
\rho \dot{e} & =\rho e_{\ominus} \dot{\Theta}+\rho e_{\rho} \dot{\rho}+\rho e_{\mathrm{q}_{e}} \cdot \mathbf{q}_{e}+\rho e_{\left\langle\Pi_{e}\right\rangle} \cdot \overline{\left\langle\boldsymbol{\Pi}_{e}\right\rangle}+\rho e_{p_{e}} \dot{p}_{e} \\
& =\rho e_{\ominus} \dot{\Theta}+\rho^{2}\left(\Theta \Psi_{\rho \Theta}-\Psi_{\rho}\right) \nabla \cdot \mathbf{v}+\rho e_{\mathrm{q}_{e}} \cdot \dot{\mathbf{q}}_{e}+\rho e_{\left\langle\Pi_{e}\right\rangle} \cdot \dot{\left\langle\boldsymbol{\Pi}_{e}\right\rangle}+\rho e_{p_{e}} \dot{p}_{e} \\
& =\rho e_{\ominus} \dot{\Theta}+\left(\rho^{2} \Theta \Psi_{\rho \Theta}-p\right) \nabla \cdot \mathbf{v}+\rho e_{\mathrm{q}_{e}} \cdot \dot{\mathbf{q}}_{e}+\rho e_{\left\langle\Pi_{e}\right\rangle} \cdot \dot{\left\langle\boldsymbol{\Pi}_{e}\right\rangle}+\rho e_{p_{e}} \dot{p}_{e}
\end{aligned}
$$

where

$$
\rho e_{\Theta}=-\rho \Theta \bar{\Psi}_{\Theta \Theta}-\frac{\theta}{2}\left(\left(\frac{\tau_{q}}{k_{e} \Theta}\right)_{\Theta \Theta}\left|\mathbf{q}_{e}\right|^{2}+\left(\frac{\tau_{\pi}}{2 \mu_{e}}\right)_{\Theta \Theta}\left|\left\langle\boldsymbol{\Pi}_{e}\right\rangle\right|^{2}+\left(\frac{\tau_{\pi}}{\zeta_{e}}\right)_{\Theta \Theta} p_{e}^{2}\right)
$$

and the positive equilibrium heat capacity $-\Theta \bar{\Psi}_{\ominus \ominus}$ is recoverable whenever the previous ratios are constant or show a linear dependence on $\Theta$.

Therefore, within the constitutive theory of this section, the governing system reads as follows

$$
\begin{aligned}
& \dot{\rho}+\rho \nabla \cdot \mathbf{v}=0, \\
& \rho \dot{\mathbf{v}}+p_{\rho} \nabla \rho+\rho^{2} \Psi_{\Theta \rho} \nabla \Theta-\mu_{s} \Delta \mathbf{v}-\left(\zeta_{s}+\frac{1}{3} \mu_{s}\right) \nabla \nabla \cdot \mathbf{v}-\nabla \cdot\left\langle\boldsymbol{\Pi}_{e}\right\rangle+\nabla p_{e}+\mu_{D} R \mathbf{v}-\rho \mathbf{b}=2 \mathbf{D} \nabla \mu_{s} \\
& +\nabla \cdot \mathbf{v} \nabla\left(\zeta_{s}-\frac{2}{3} \mu_{s}\right)-\nabla \mathbf{q}_{e}^{T} p_{\mathrm{q}_{e}}-\nabla\left\langle\boldsymbol{\Pi}_{e}\right\rangle^{T} p_{\left\langle\Pi_{e}\right\rangle}-p_{p_{e}} \nabla p_{e} \\
& \rho e_{\Theta} \dot{\Theta}+\rho^{2} \Theta \psi_{\Theta \rho} \nabla \cdot \mathbf{v}-k_{F} \Delta \Theta+\nabla \cdot \mathbf{q}_{e}-\rho r=-\rho e_{q_{e}} \cdot \dot{\mathbf{q}}_{e}-\rho e_{\left\langle\Pi_{e}\right\rangle} \cdot \overline{\left\langle\boldsymbol{\Pi}_{e}\right\rangle}-\rho e_{p_{e}} \dot{p}_{e}+\nabla k_{F} \cdot \nabla \Theta \\
& +2 \mu_{s}|\langle\mathbf{D}\rangle|^{2}+\zeta_{s}|\nabla \cdot \mathbf{v}|^{2}+\left\langle\boldsymbol{\Pi}_{e}\right\rangle \cdot\langle\mathbf{D}\rangle-p_{e} \nabla \cdot \mathbf{v}, \\
& \tau_{q}\left(\dot{q}_{e}-\mathbf{W} \mathbf{q}_{e}\right)+\mathbf{q}_{e}=-k_{e} \nabla \Theta, \\
& \tau_{\pi}\left(\overline{\left\langle\boldsymbol{\Pi}_{e}\right\rangle}-\mathbf{W}\left\langle\boldsymbol{\Pi}_{e}\right\rangle+\left\langle\boldsymbol{\Pi}_{e}\right\rangle \mathbf{W}\right)+\left\langle\boldsymbol{\Pi}_{e}\right\rangle=2 \mu_{e}\langle\mathbf{D}\rangle . \\
& \tau_{\pi} \dot{p}_{e}+p_{e}=-\zeta_{e} \nabla \cdot \mathbf{v} .
\end{aligned}
$$

In order that the nonlinear parabolic heat equation (4.4) don't degenerate and yields well-posed problems, we need to assume the heat capacity $e_{\ominus}>0$, as suggested by physical arguments.

Even if we restrict our discussion to constant parameters, we obtain a coupled system between heat and momentum equations, which might capture the main properties of a non-isothermal viscoelastic polymeric fluid in a porous context, where noninstantaneous/lagging and dissipation effects combine together. However, in the absence of the dissipation mechanisms, due to the presence of diffusion terms, our model might become a suitable hyperbolic model for the cells within chemotaxis. The idea of hyperbolic modelling in this framework, even preserving the Keller-Segel reaction-diffusion equation for the chemical concentration [8], has been already performed in [9].

System (4.2)-(4.7) is a new system to be investigated mathematically under suitable initial and boundary conditions. It may also model a thermal/penetrative convection problem in a horizontal layer $\mathbb{R}^{2} \times(0, d)$, heated from below, within the Boussinesq approximation and, in our opinion, it may become the starting model to understand the basic processes in blood capillary growth and astrophysical settings.

Indeed we may gradually make it more and more sophisticated and "hopefully" more realistic, by the additional contribution on the right-hand side of the momentum equation of (self-gravity/concentration) gradient forces, so to describe both chemotactic phenomena and gravitational instability mechanisms. Of course, the system should be closed with a parabolic reaction-diffusion equation for the self-gravity potential/chemoattractant concentration of Keller-Segel type. 
In the isothermal case, our model reduces to the form

$$
\begin{aligned}
& \dot{\rho}+\rho \nabla \cdot \mathbf{v}=0 \\
& \begin{aligned}
\rho \dot{\mathbf{v}} & =p_{\rho} \nabla \rho-\mu_{s} \Delta \mathbf{v}-\left(\zeta_{s}+\frac{1}{3} \mu_{s}\right) \nabla \nabla \cdot \mathbf{v}-\nabla \cdot\left\langle\boldsymbol{\Pi}_{e}\right\rangle+\nabla p_{e}+\mu_{D} R \mathbf{v}-\rho \mathbf{b}=2 \mathbf{D} \nabla \mu_{s}+\nabla \cdot \mathbf{v} \nabla\left(\zeta_{s}-\frac{2}{3} \mu_{s}\right) \\
& -\nabla\left\langle\boldsymbol{\Pi}_{e}\right\rangle^{T} p_{\left\langle\Pi_{e}\right\rangle}-p_{p_{e}} \nabla p_{e}
\end{aligned} \\
& \\
& \tau_{\pi}\left(\overline{\dot{\left.\boldsymbol{\Pi}_{e}\right\rangle}}-\mathbf{W}\left\langle\boldsymbol{\Pi}_{e}\right\rangle+\left\langle\boldsymbol{\Pi}_{e}\right\rangle \mathbf{W}\right)+\left\langle\boldsymbol{\Pi}_{e}\right\rangle=2 \mu_{e}\langle\mathbf{D}\rangle \\
& \tau_{\pi} \dot{p}_{e}+p_{e}=-\zeta_{e} \nabla \cdot \mathbf{v}
\end{aligned}
$$

and may be used for modelling a high molecular weight fluid, in order to fit a dilute polymeric solution composed of a Newtonian solvent and a polymeric solution with more accuracy (for example confined in microtubes [33, 34, 21]).

Meanwhile, in the inviscid case, the evolution system reads

$$
\begin{aligned}
& \dot{\rho}+\rho \nabla \cdot \mathbf{v}=0, \\
& \rho \dot{\mathbf{v}}+p_{\rho} \nabla \rho+p_{\Theta} \nabla \Theta+\mu_{D} R \mathbf{v}-\rho \mathbf{b}=-\nabla \mathbf{q}_{e}^{T} p_{\mathrm{qe}} \\
& \rho e_{\Theta} \dot{\Theta}+\rho^{2} \Theta \Psi_{\Theta \rho} \nabla \cdot \mathbf{v}-k_{F} \Delta \Theta+\nabla \cdot \mathbf{q}_{e}-\rho r \\
& \quad=-\rho e_{\mathrm{q}_{e}} \cdot \dot{\mathbf{q}}_{e}+\nabla k_{F} \cdot \nabla \Theta, \\
& \tau_{q}\left(\dot{\mathbf{q}}_{e}-\mathbf{W} \mathbf{q}_{e}\right)+\mathbf{q}_{e}=-k_{e} \nabla \Theta,
\end{aligned}
$$

and furnishes a nonlinear non-Fourier heat conduction theory for a barotropic gas in a porous medium which generalizes the Cattaneo-Christov model [27] and might be suitable to model the non classical thermal effects in an astrophysical setting. For example in [35], where the heat transfer mechanisms in solar flares are investigated, it is proven that it is "absolutely necessary" to take into account the effects of the collisional relaxation of the heat flux.

\section{The linearized model: a spectral analysis tool}

As a starting point we choose a quiescent homogeneous state, described by $\mathbf{v}=\mathbf{0}, \rho=\rho_{0}$ positive and constant, $\Theta=\Theta_{0}$ positive and constant, $\mathbf{q}_{e}=\mathbf{0},\left\langle\boldsymbol{\Pi}_{e}\right\rangle=\mathbf{0}$ and $p_{e}=0$.

In order to better understand the strengthness of our modelling, we now linearize system (4.2)-(4.7) around the above equilibrium solution, by introducing a perturbation state $\delta^{*} s$, given by $\delta^{*} \mathbf{v}=\mathbf{v}, \delta^{*} \rho, \delta^{*} \Theta, \delta^{*} \mathbf{q}_{e}=\mathbf{q}_{e}, \delta^{*}\left\langle\boldsymbol{\Pi}_{e}\right\rangle=\left\langle\boldsymbol{\Pi}_{e}\right\rangle$ and $\delta^{*} p_{e}=p_{e}$.

For small perturbations, we easily obtain the linearized system

$$
\begin{aligned}
\delta^{*} \rho_{t}= & -\rho_{0} \nabla \cdot \delta^{*} \mathbf{v} \\
\rho_{0} \delta^{*} \mathbf{v}_{t}= & +\mu_{s}(0) \Delta \delta^{*} \mathbf{v}+\left(\zeta_{s}(0)+\frac{1}{3} \mu_{s}(0)\right) \nabla\left(\nabla \cdot \delta^{*} \mathbf{v}\right)-\mu_{D} R \delta^{*} \mathbf{v} \\
& -p_{\rho}(0) \nabla \delta^{*} \rho-\rho_{0}^{2} \Psi_{\rho \Theta}(0) \nabla \delta^{*} \Theta+\nabla \cdot \delta^{*}\left\langle\boldsymbol{\Pi}_{e}\right\rangle-\nabla \delta^{*} p_{e}, \\
\rho_{0} e_{\Theta}(0) \delta^{*} \Theta_{t}= & -\rho_{0} \Theta_{0} \Psi_{\Theta \rho}(0) \nabla \cdot \delta^{*} \mathbf{v}+k_{F}(0) \Delta \delta^{*} \Theta-\nabla \cdot \delta^{*} \mathbf{q}_{e} \\
\tau_{\pi}(0) \delta^{*}\left\langle\boldsymbol{\Pi}_{e}\right\rangle_{t}= & -\delta^{*}\left\langle\boldsymbol{\Pi}_{e}\right\rangle+\mu_{e}(0)\left[\nabla \delta^{*} \mathbf{v}+\nabla \delta^{*} \mathbf{v}^{T}\right]-\frac{2}{3} \mu_{e}(0) \nabla \cdot \delta^{*} \mathbf{v} \mathbb{I} \\
\tau_{\pi}(0) \delta^{*} p_{e t}= & -\delta^{*} p_{e}-\zeta_{e}(0) \nabla \cdot \delta^{*} \mathbf{v} \\
\tau_{q}(0) \delta^{*} \mathbf{q}_{e t}= & -\delta^{*} \mathbf{q}_{e}-k_{e}(0) \nabla \delta^{*} \Theta
\end{aligned}
$$

where we have used the notation (0), to denote the constancy of previous parameters as evaluated at equilibrium state.

As it is customary in linear stability analysis, we introduce a spectral tool in order to investigate the behavior of these perturbations. So, in spatially unbounded domain, we address to the Fourier modes technique, and look for perturbations of the dispersive wave form

$$
0 \neq \delta^{*} s=s_{1} e^{i(k \cdot x-\omega t)}
$$

where $s_{1}$ is the constant amplitude, $\mathbf{k}$ is the (real) wave number vector, with $k^{2}>0$, and $\omega$ denotes the frequency of the disturbance. 
In the sequel, for simplicity, we omit the notation (0), without misunderstanding. By using standard identities, from (5.1) we easily get the linear algebraic system for the perturbation amplitude $s_{1}=\left(\rho_{1}, v_{1}, \Theta_{1}, \Pi_{1}, p_{1}, q_{1}\right)$

$$
\begin{aligned}
& \omega \rho_{1}-\rho_{0} \mathbf{v}_{1} \cdot \mathbf{k}=0, \\
& \rho_{0} \omega \mathbf{v}_{1}=C_{s}^{2} \rho_{1} \mathbf{k}+\rho_{0}^{2} \bar{\Psi}_{\rho \ominus} \Theta_{1} \mathbf{k}-i \mu_{s} k^{2} \mathbf{v}_{1}-i\left(\zeta_{s}+\frac{1}{3} \mu_{s}\right)\left(\mathbf{v}_{1} \cdot \mathbf{k}\right) \mathbf{k}-i \mu_{D} R \mathbf{v}_{1}-\Pi_{1} \mathbf{k}+p_{1} \mathbf{k}, \\
& \rho_{0} e_{\ominus} \omega \Theta_{1}+\rho_{0}^{2} \Theta_{0} \bar{\Psi}_{\Theta \rho} \mathbf{v}_{1} \cdot \mathbf{k}=-i k_{F} \Theta_{1} k^{2}+\mathbf{q}_{1} \cdot \mathbf{k}, \\
& \left(\tau_{\pi} \omega+i\right) \boldsymbol{\Pi}_{1}=-\mu_{e}\left(\mathbf{v}_{1} \otimes \mathbf{k}+\mathbf{k} \otimes \mathbf{v}_{1}-\frac{2}{3} \mathbf{v}_{1} \cdot \mathbf{k} \mathbb{I}\right), \\
& \left(\tau_{\pi} \omega+i\right) p_{1}=\zeta_{e} \mathbf{v}_{1} \cdot \mathbf{k}, \\
& \left(\tau_{q} \omega+i\right) \mathbf{q}_{1}=k_{e} \Theta_{1} \mathbf{k},
\end{aligned}
$$

where, for brevity, we have simplified the notations, as follows

$$
\boldsymbol{\Pi}_{1}=\langle\boldsymbol{\Pi}\rangle_{1}, p_{1}=p_{e 1}, \mathbf{q}_{1}=\mathbf{q}_{e 1}
$$

and $C_{s}^{2}$ is now the isothermal sound speed. Moreover, from (4.1), we have $e_{\ominus}=-\Theta_{0} \bar{\Psi}_{\ominus \ominus}$, and hence, since phenomenological observations suggest $e_{\Theta}>0$, it is likely that $\bar{\Psi}_{\ominus \ominus}<0$.

The momentum equation (5.2) $)_{2}$ and the thermal constitutive equation (5.2) 6 may be resolved in normal $\left(\mathbf{n}=\frac{k}{k}\right.$, parallel to the wave number vector) and tangential components along two orthogonal transverse directions $\mathbf{t}_{1}$ and $\mathbf{t}_{2}$, by using the decomposition formula

For $\omega \neq 0$, from $(5.2)_{1}$ we derive the link

$$
\mathbf{u}=\left(\mathbf{u} \cdot \frac{\mathbf{k}}{k}\right) \frac{\mathbf{k}}{k}+\left(\mathbf{u} \cdot \mathbf{t}_{1}\right) \mathbf{t}_{1}+\left(\mathbf{u} \cdot \mathbf{t}_{2}\right) \mathbf{t}_{2}
$$

$$
\rho_{1}=\frac{\rho_{0}}{\omega} \mathbf{v}_{1} \cdot \mathbf{k}
$$

whereas for $\tau_{q} \omega+i \neq 0$, from $(5.2)_{6}$ we find

$$
\mathbf{q}_{1} \cdot \mathbf{k}=\frac{k_{e}}{\tau_{q} \omega+i} \Theta_{1} k^{2}, \quad \mathbf{q}_{1} \cdot \mathbf{t}_{i}=0, i=1,2 .
$$

Finally, for $\tau_{\pi} \omega+i \neq 0$, from (5.2) 4,5 , we easily recover the new relations

$$
\begin{gathered}
\boldsymbol{\Pi}_{1} \mathbf{k} \cdot \mathbf{k}=-\frac{4}{3} \frac{\mu_{e} k^{2}}{\tau_{\pi} \omega+i} \mathbf{v}_{1} \cdot \mathbf{k}, \quad \boldsymbol{\Pi}_{1} \mathbf{k} \cdot \mathbf{t}_{i}=-\frac{\mu_{e} k^{2}}{\tau_{\pi} \omega+i} \mathbf{v}_{1} \cdot \mathbf{t}_{i}, \quad i=1,2, \\
p_{1}=\frac{\zeta_{e}}{\tau_{\pi} \omega+i} \mathbf{v}_{1} \cdot \mathbf{k} .
\end{gathered}
$$

Let now $\omega \notin\left\{0,-\frac{i}{\tau_{q}},-\frac{i}{\tau_{\pi}}\right\}$; by gathering the previous relations (5.3)-(5.6), we reduce the dispersion system (5.2) to the following Cramer form in the essential amplitudes $\mathbf{v}_{1} \cdot \mathbf{n}, \mathbf{v}_{1} \cdot \mathbf{t}_{i},(i=1,2)$ and $\Theta_{1}$

$$
\begin{aligned}
& {\left[\omega^{2}-C_{s}^{2} k^{2}+i \omega k^{2}\left(\frac{\zeta_{s}}{\rho_{0}}+\frac{4}{3} \nu_{s}\right)+\nu_{D} R i \omega-\frac{\omega k^{2}}{\tau_{\pi} \omega+i}\left(\frac{\zeta_{e}}{\rho_{0}}+\frac{4}{3} \nu_{e}\right)\right] \mathbf{v}_{1} \cdot \mathbf{n}-\rho_{0} \bar{\Psi}_{\rho \ominus \omega k} \Theta_{1}=0,} \\
& \left(\omega+i \nu_{s} k^{2}+\nu_{D} R i-\frac{\nu_{e} k^{2}}{\tau_{\pi} \omega+i}\right) \mathbf{v}_{1} \cdot \mathbf{t}_{i}=0, \quad i=1,2, \\
& \frac{\rho_{0} \Theta_{0} \bar{\Psi}_{\ominus \rho}}{e_{\ominus}} \mathbf{v}_{1} \cdot \mathbf{n}+\left(\frac{\omega}{k}+i \chi_{F} k-\frac{\chi_{e} k}{\tau_{q} \omega+i}\right) \Theta_{1}=0,
\end{aligned}
$$

where we have still used the well-known notations for the kinematical viscosity $\nu=\mu / \rho$, whereas $\chi=k / \rho e_{\ominus}$ stands for the classical thermal diffusivity.

By applying the Cramer's rule on system (5.7), the general dispersion relation follows straightaway

$$
\begin{array}{r}
\left\{\left[\omega^{2}-C_{s}^{2} k^{2}+i \omega k^{2}\left(\frac{\zeta_{s}}{\rho_{0}}+\frac{4}{3} \nu_{s}\right)+\nu_{D} R i \omega-\frac{\omega k^{2}}{\tau_{\pi} \omega+i}\left(\frac{\zeta_{e}}{\rho_{0}}+\frac{4}{3} \nu_{e}\right)\right] \times\left(\frac{\omega}{k}+i \chi_{F} k-\frac{\chi_{e} k}{\tau_{q} \omega+i}\right)+\frac{\left.\rho_{0}^{2} \Theta_{0} \bar{\Psi}_{\ominus \rho}^{2} \omega k\right\} \times}{e_{\ominus}} \times\right. \\
\times\left(\omega+i \nu_{s} k^{2}+\nu_{D} R i-\frac{\nu_{e} k^{2}}{\tau_{\pi} \omega+i}\right)^{2}=0 .
\end{array}
$$

As $\bar{\Psi}_{\Theta \ominus}<0$, the parameter $k_{1}$, defined as in [27] by

may be henceforth considered negative.

$$
k_{1}=\frac{\rho_{0}^{2} \bar{\Psi}_{\ominus \rho}^{2}}{\bar{\Psi}_{\ominus \ominus}}
$$

Now we are going in performing a detailed discussion of the dispersion equation (5.8) in some significant limit cases, having in mind future applications of our modelling. 
5.1. The linearized isothermal J-S viscoelatic fluid in a porous medium: viscoelastic-poroacoustic dispersive waves

In this case (5.8) leads to the following separate dispersion relations

$$
\begin{aligned}
& \left(\omega+i \nu_{s} k^{2}+\nu_{D} R i-\frac{\nu_{e} k^{2}}{\tau_{\pi} \omega+i}\right)^{2}=0, \\
& \omega^{2}-C_{s}^{2} k^{2}+i \omega k^{2}\left(\frac{\zeta_{s}}{\rho_{0}}+\frac{4}{3} \nu_{s}\right)+\nu_{D} R i \omega-\frac{\omega k^{2}}{\tau_{\pi} \omega+i}\left(\frac{\zeta_{e}}{\rho_{0}}+\frac{4}{3} \nu_{e}\right)=0,
\end{aligned}
$$

which account for transverse and longitudinal modes, respectively.

Setting

$$
C_{V T}^{2}=\frac{\nu_{s}+\nu_{e}}{\tau_{\pi}}, \quad C_{V L}^{2}=\frac{\zeta_{s}+\zeta_{e}}{\rho_{0} \tau_{\pi}}+\frac{4}{3} \frac{\nu_{s}+\nu_{e}}{\tau_{\pi}},
$$

it is an easy matter to rewrite the previous dispersion relations (5.9) in terms of the growth rate parameter $\sigma=-i \omega$ :

$$
\begin{aligned}
& \sigma^{2}+\sigma\left(\frac{1}{\tau_{\pi}}+\nu_{s} k^{2}+\nu_{D} R\right)+C_{V T}^{2} k^{2}+\frac{\nu_{D} R}{\tau_{\pi}}=0, \\
& \sigma^{3}+\sigma^{2}\left(\frac{1}{\tau_{\pi}}+\frac{\zeta_{s}}{\rho_{0}} k^{2}+\frac{4 \nu_{s}}{3} k^{2}+\nu_{D} R\right)+\sigma\left(C_{V L}^{2} k^{2}+C_{S}^{2} k^{2}+\frac{\nu_{D} R}{\tau_{\pi}}\right)+\frac{C_{S}^{2} k^{2}}{\tau_{\pi}}=0,
\end{aligned}
$$

At a first sight, it may be emphasized the jointly damping effects of the porous and solvent terms. Even in the absence of the solvent component (hyperbolic model), the presence of the Darcy term changes the behavior of both transverse and longitudinal viscoelastic-poroacoustic waves, but it is worth to note that the diffusion term strengthens the stabilizing effect.

With regard the cubic equation $(5.10)_{2}$, by applying the Routh-Hurwitz stability test, we may establish the asymptotic stability of the equilibrium state. As a matter of fact, we know that the solutions to $(5.10)_{2}$ have negative real part (and this guarantees the asymptotic stability of the modes) if and only if all the coefficients are positive and the following condition

$$
\left(\frac{1}{\tau_{\pi}}+\frac{\zeta_{s}}{\rho_{0}} k^{2}+\frac{4 \nu_{s}}{3} k^{2}+\nu_{D} R\right)\left(C_{V L}^{2} k^{2}+C_{S}^{2} k^{2}+\frac{\nu_{D} R}{\tau_{\pi}}\right)-\frac{C_{S}^{2} k^{2}}{\tau_{\pi}}>0
$$

holds. In our case this condition is trivially satisfied.

In the hyperbolic case $\left(\nu_{s}=\zeta_{s}=0\right)$ the purely oscillatory regimes are lost, and hence we always obtain (fast and slow) damped longitudinal viscoleastic-poroacoustic dispersive waves.

Transverse modes are now investigated by solving the quadratic equation (5.10) $)_{1}$; meanwhile in the incompressible case it is the unique dispersion relation to be commented.

In the absence of the porous term and for a purely polymeric melt $\left(\nu_{D}=\nu_{s}=0\right)$, the quadratic equation simplifies to

$$
\tau_{\pi} \sigma^{2}+\sigma+\nu k^{2}=0
$$

which admits the solutions

$$
\sigma^{ \pm}=-\frac{1}{2 \tau_{\pi}} \pm \sqrt{\frac{1}{4 \tau_{\pi}^{2}}-\frac{\nu}{\tau_{\pi}} k^{2}}
$$

In view of the smallness of the relaxation time $\tau_{\pi}$, the nature of $\sigma$ is generally responsible of two distinct regimes, given by transverse shear modes exhibiting a damped oscillatory behavior, so the purely oscillatory shear waves are lost. Obviously, such behavior is strengthened by the additional presence of the Darcy term.

For polymeric solutions with positive solvent viscosity $\left(\nu_{s}>0\right)$, negative elastic viscosity $\left(\nu_{e}<0\right)$ and again in absence of porous effects $\left(\nu_{D}=0\right)$, we may introduce the viscoelastic dimensionless retardation parameter $r_{\pi}$ so that

$$
\nu_{s}=r_{\pi} \nu, \quad \nu_{e}=\left(1-r_{\pi}\right) \nu, \quad \tau_{D}=\tau_{\pi} r_{\pi} .
$$

Then $(5.10)_{1}$ reads

$$
\tau_{\pi} \sigma^{2}+\sigma\left(1+\nu \tau_{D} k^{2}\right)+\nu k^{2}=0
$$

and its solutions become

$$
\sigma^{ \pm}=\frac{-1-\nu \tau_{D} k^{2} \pm \sqrt{\Delta^{*}}}{2 \tau_{\pi}}
$$

with

$$
\Delta^{*}=\nu^{2} \tau_{D}^{2} k^{4}+2 \nu k^{2}\left(\tau_{D}-2 \tau_{\pi}\right)+1 .
$$

We immediately realize that, whenever $\tau_{D}-2 \tau_{\pi} \geq 0$, we have $\Delta^{*}>0$ for any $k^{2}>0$, so that $\sigma^{ \pm}$are real and negative and this allows the exponential decay in time for any transverse Fourier modes. 
This is in agreement with the findings in [36] for the analogous dual-phase-lag heat model, within the first order Taylor approximation. Nevertheless, following the procedure of [37], in the same heat frame, we can see that the general stability discussion is strictly related to the sign of $1-r_{\pi}$. In fact, by solving the bi-quadratic equation $\Delta^{*}=0$, we find

$$
k^{2}=\frac{-\nu\left(\tau_{D}-2 \tau_{\pi}\right) \pm \sqrt{\bar{\triangle}^{*}}}{\nu^{2} \tau_{D}^{2}}
$$

with

$$
\bar{\Delta}^{*}=4 \nu^{2} \tau_{\pi}\left(\tau_{\pi}-\tau_{D}\right)=4 \nu^{2} \tau_{\pi}^{2}\left(1-r_{\pi}\right) .
$$

So, for $r_{\pi}>1$, corresponding to the empirically legitimated retarded response of $\mathbf{D}$ on $\boldsymbol{\Pi}$ (see e.g.[38]), we still recover the previous exponentially decreasing regimes, while, for $r_{\pi}<1$, besides such regimes, we also obtain damped oscillatory viscoelastic modes (transverse shear dispersive waves), accounting for the stabilizing presence of the Newtonian stress.

In conclusion, the equilibrium solution is always asymptotically linearly stable independently on the sign $1-r_{\pi}$, contrarily to the nonlinear stability results proven in [19], where, by using energy arguments, we showed that asymptotic nonlinear stability may be recovered only in the range $1<r_{\pi}<\frac{3}{2}$.

\subsection{The linearized inviscid case: thermo-poroacoustic dispersive waves}

In this context, relation (5.8) reduces to the form

$$
\left(\omega+\nu_{D} R i\right)^{2}\left[\left(\omega^{2}-C_{s}^{2} k^{2}+\nu_{D} R i \omega\right)\left(\frac{\omega}{k}+i \chi_{F} k-\frac{\chi_{e} k}{\tau_{q} \omega+i}\right)-\left|k_{1}\right| \omega k\right]=0 .
$$

From (5.12) we firstly derive a stable mode due to the presence of the Darcy term with the frequency

$$
\omega=-i \nu_{D} R
$$

but also the general dispersion equation

$$
\left(\omega^{2}-C_{s}^{2} k^{2}+\nu_{D} R i \omega\right)\left(\frac{\omega}{k}+i \chi_{F} k-\frac{\chi_{e} k}{\tau_{q} \omega+i}\right)=\left|k_{1}\right| \omega k
$$

which accounts for the coupled thermo-poroacoustic effects.

It is worth to note that, when the coupling parameter $k_{1}$ vanishes and also $\nu_{D}=0$, acoustic and thermal responses are completely separate.

The split form of (5.13) gives

$$
\omega^{2}-C_{s}^{2} k^{2}=0, \quad \tau_{q} \omega^{2}+i \omega\left(1+\chi_{F} k^{2} \tau_{q}\right)-\left(\chi_{F}+\chi_{e}\right) k^{2}=0 .
$$

From (5.14) $)_{1}$ we find the stable modes, corresponding to oscillatory acoustic dispersive waves. Then, if we introduce the thermal dimensionless retardation parameter $r_{q}$ and pose

$$
k_{F}=r_{q} k, \quad k_{e}=\left(1-r_{q}\right) k, \quad \tau_{\ominus}=\tau_{q} r_{q},
$$

$(5.14)_{2}$ yields the following equation

$$
\tau_{q} \sigma^{2}+\sigma\left(1+\chi \tau_{\ominus} k^{2}\right)+\chi k^{2}=0 .
$$

Since it is formally similar to (5.11), the linear stability analysis follows straightaway. In particular, the asymptotic stability is always recoverable also in the case $r_{\Theta}>1$, namely for $\tau_{\Theta}>\tau_{q}$, describing the retarded response of $\nabla \Theta$ on $\mathbf{q}$, which is experimentally possible even if in disagreement with the causality principle (see e.g. [23]).

Within the incompressible setting, we find only transverse thermo-poro dispersive waves, accounting for the dissipative effects due to the simultaneous presence of the porous and Fourier terms.

We now focus our attention on the general dispersion equation (5.13).

In absence of porous effects, it is a simple matter to rewrite it as the following quartic equation in $\sigma$

$$
\sigma^{4}+\sigma^{3}\left(\frac{1}{\tau_{q}}+\chi_{F} k^{2}\right)+\sigma^{2}\left(C_{s}^{2}+C_{T}^{2}+\left|k_{1}\right|\right) k^{2}+\sigma\left[C_{s}^{2}\left(\chi_{F} k^{2}+\frac{1}{\tau_{q}}\right)+\frac{\left|k_{1}\right|}{\tau_{q}}\right] k^{2}+C_{s}^{2} C_{T}^{2} k^{4}=0,
$$

where we have defined $C_{T}^{2}=\frac{\chi_{e}+\chi_{e}}{\tau_{q}}$.

When $\chi_{F}=0$, the model becomes hyperbolic and $C_{T}^{2}$ represents the square of the thermal wave speed in the absence of compressional effects, which is typical of the non-Fourier hyperbolic models. It is worth to note that, in this case, equation (5.16) accounts for coupled (fast and slow) thermo-acoustic longitudinal dispersive waves, which in turn may be compared with the analogous longitudinal waves, investigated in [27] within the nonlinear wave propagation frame. 
Nevertheless, in our linear framework and, analogously to the viscoelastic setting, purely oscillatory (transverse and longitudinal) dispersive waves are lost.

By employing the Routh-Hurwitz stability criterion, equation (5.16) allows for recovering the exponentially asymptotic stability of the homogeneous equilibrium state, regardless of the sign of $\left(1-r_{q}\right)$. In fact, its solutions have negative real part (corresponding to four asymptotically stable modes) if and only if, besides the positivity of the coefficients, the further conditions

$$
\begin{aligned}
& \left(1+\tau_{q} \chi_{F} k^{2}\right)\left(C_{s}^{2}+C_{T}^{2}+\left|k_{1}\right|\right)-\left[C_{s}^{2}\left(1+\tau_{q} \chi_{F} k^{2}\right)+\left|k_{1}\right|\right]>0 \\
& \left(1+\tau_{q} \chi_{F} k^{2}\right)\left(C_{s}^{2}+C_{T}^{2}+\left|k_{1}\right|\right)\left[C_{s}^{2}\left(1+\tau_{q} \chi_{F} k^{2}\right)+\left|k_{1}\right|\right]-\left(1+\tau_{q} \chi_{F} k^{2}\right)^{2} C_{s}^{2} C_{T}^{2}-\left[C_{s}^{2}\left(1+\tau_{q} \chi_{F} k^{2}\right)+\left|k_{1}\right|\right]^{2}>0
\end{aligned}
$$

hold. The validity of these conditions can be trivially checked.

\subsection{A link with the non-isothermal Kelvin-Voight viscoelasticity}

It is noteworthy that, only within the linear theory, by employing the phase-lag concept together with "backwards" temporal shiftings, a Kelvin-Voight viscoelastic model is always derivable from a Jeffreys-type viscoelasticity (see e.g. [4]). This is strictly related to the fact that a single-phase-lag theory is always recoverable from a dual-phase-lag theory if one works with a difference delay parameter.

In our setting the difference delay parameter is just given by $\tau_{\pi}\left(1-r_{\pi}\right)$; so, up to a first-order Taylor approximation, we recover a viscoelastic model of Walters-B type when $r_{\pi}<1$, whereas the linear viscoelastic theory of Rivlin-Ericksen type is obtained for $r_{\pi}>1$.

For polymeric liquids the new constitutive equation reads

$$
\boldsymbol{\Pi}=2 \mu\left[\mathbf{D}-\tau_{\pi}\left(1-r_{\pi}\right) \frac{\partial}{\partial t} \mathbf{D}\right]
$$

which may be further generalized to account for compressional terms.

In spatially unbounded domains and in a complete agreement with the results concerning the phase-lag heat theories [37], we can show that the retarded response of $\mathbf{D}$ on $\boldsymbol{\Pi}$ is always stabilizing, on the contrary the advanced behavior leads to the onset of viscoelastic instabilities.

Following the same linear stability tool as before, but starting from equation (5.17), we obtain the linearized perturbation system

$$
\begin{aligned}
\nabla \cdot \delta^{*} \mathbf{v} & =0 \\
\delta^{*} \mathbf{v}_{t} & =-\frac{1}{\rho} \nabla \delta^{*} p+\nu \Delta \delta^{*} \mathbf{v}-\nu \tau_{\pi}\left(1-r_{\pi}\right) \Delta \delta^{*} \mathbf{v}_{t}-\nu_{D} R \delta^{*} \mathbf{v}
\end{aligned}
$$

Looking for Fourier modes perturbations, we easily get the simplified dispersion system

$$
\begin{aligned}
& \mathbf{v}_{1} \cdot \mathbf{k}=0 \\
& \sigma \mathbf{v}_{1}=-\frac{p_{1}}{\rho} i \mathbf{k}+\nu\left[-1+\tau_{\pi}\left(1-r_{\pi}\right) \sigma\right] k^{2} \mathbf{v}_{1}-\nu_{D} R \mathbf{v}_{1}
\end{aligned}
$$

where $p_{1}$ is now the amplitude relative to the pressure $p$.

In view of the constraint $\mathbf{v}_{1} \cdot \mathbf{k}=0$, we find only transverse modes and, by solving the final dispersion relation, we have

$$
\sigma=-\frac{\nu k^{2}+\nu_{D} R}{1-\nu \tau_{\pi}\left(1-r_{\pi}\right) k^{2}}
$$

As we can see, the stability discussion is affected by the sign of $1-r_{\pi}$. Hence, for $r_{\pi}>1$, we always obtain exponentially asymptotic stability and the Darcy term plays the same role as a cooling effect; on the other hand, for $r_{\pi}<1$, we always have instability.

\section{Final comments}

We have investigated the constitutive theory for a new type of thermoviscoelastic polymeric fluid in a porous medium; linear stability results and rheological analogies with different topics, recommend its applicability in various settings of physics, astrophysics and biology.

In particular, the characteristics of our hydrodynamical model may fit both chemotactic and gravitational instability mechanisms; in these contexts, the choice of the additional evolution equation for the chemoattractant and for the self-gravity potential turns out to be a challenge issue. Indeed, in these research areas the further presence of a magnetic field is unlikely 
to be neglected. To this purpose it is however worth to put into evidence that the coupling between magnetic fields and porous media should be carefully dealt with caution especially when investigating convection problems (see e.g. [39, 40]).

Besides, numerical simulations indicate that the propagation of incompressible torsional Alfvén waves on vertical flux tubes be the driving mechanism in generating complex network patterns; so, by comparison, we may conjecture the same ability for the formally similar incompressible torsional shear viscoelastic waves.

For these reasons, following the arguments of [5], we expect that our (isothermal or not) non-Newtonian environment in porous media, might describe chemotaxis better than previous hydrodynamical modellings.

Moreover, the interlacing between dissipative and relaxation behaviors both for thermal and mechanical aspects, appears to be theoretically and experimentally useful, also in connection with the instability of self-gravitating interstellar gas clouds.

In conclusion, throughout this paper we emphasize two relevant interplay effects: firstly, the interchange between diffusion and elastic properties for mechanical and thermal effects, related to parabolic and hyperbolic modellings; secondly the joint relation between micro and macro-scales events in any setting, which, in turn, lead to the important connection between kinetic and continuum theories. In effect the kinetic approach describes not only the micro-scale cellular context, but it becomes an interesting driver for the related continuum frame.

\section{Acknowledgment}

We would like to thank the referees for their helpful suggestions.

Research performed under the auspices of G.N.F.M. - I.N.d.A.M. and partially supported by Italian M.I.U.R.

\section{References}

1. Straughan B. Stability and Wave Motion in Porous Media Stability and Wave Motion in Porous Media Stability and Wave Motion Stability and Wave Motion in Porous Media, Applied Mathematical Sciences, vol. 165. Springer New York, 2008, doi: 10.1007/978-0-387-76543-3. URL http://link. springer.com/book/10.1007/978-0-387-76543-3.

2. Nield D, Bejan A. Convection in Porous Media. Springer New York, 2006, doi:10.1007/0-387-33431-9. URL http://link. springer. com/book/10.1007/0-387-33431-9.

3. Tzou D. A unified approach for heat conduction from macro to micro-scales. Int. J. Heat Mass Tran. 1995; 9:686-693, doi: 10.1115/1.2822329. URL http://dx.doi.org/10.1115/1.2822329.

4. Bird R, Armstrong R, Hassager O. Dynamics of polymeric fluids., vol. 1. Fluid Mechanics. Jhon Wiley \& Son: New York, 1987.

5. Chavanis P, Sire C. Jeans type analysis of chemotactic collapse. Physica A 2008; 387(16):4033-4052, doi:10.1016/j.physa.2008.02.025. URL http://dx.doi.org/10.1016/j.physa.2008.02.025

6. Ambrosi D, Gamba A, Serini G. Cell directional and chemotaxis in vascular morphogenesis. Bull. Math. Biol. 2004; 66(6):1851-1873, doi:10.1016/j.bulm.2004.04.004. URL http://dx.doi.org/10.1016/j.bulm.2004.04.004.

7. Dolak Y, Hillen T. Cattaneo models for chemosensitive movement. numerical solution and pattern formation. J. Math. Biol. 2003; 46:461-478, doi:10.1007/s00285-003-0221-y. URL http://dx.doi.org/10.1007/s00285-003-0221-y.

8. Keller E, Segel L. Initiation of slime mold aggregation viewed as an instability. J. Theor. Biol. 1970; 26(3):399-415, doi: 10.1016/0022-5193(70)90092-5. URL http://dx.doi.org/10.1016/0022-5193(70)90092-5.

9. Bellomo N, Bellouquid A, Nieto J, Soler J. Multiscale biological tissue models and flux-limited chemotaxis for multicellular growing systems. Math. Models Methods Appl. Sci. 2010; 20(7):1179-1207, doi:10.1142/S0218202510004568. URL http://dx.doi.org/10. 1142/S0218202510004568.

10. Priest E, Forbes T. Magnetic reconnection: MHD theory and applications. Cambridge University Press, 2007.

11. Jess D, Mathioudakis M, Erdélyi R, Crockett P, Keenan F, Christian D. Alfvèn waves in the lower solar atmosphere. Science 2009; 323(5921):1582-1585, doi:10.1126/science.1168680. URL http://dx.doi.org/10.1126/science.1168680.

12. Antolin P, Shibata K. The role of torsional Alfvén waves in coronal heating. Astrophys. J. 2010; 712(1):494-510, doi:10.1088/ 0004-637X/712/1/494. URL http://dx.doi.org/10.1088/0004-637X/712/1/494.

13. Fedun V, Shelyag S, Verth G, Mathioudakis M, Erdélyi R. Mhd waves generated by high-frequency photospheric vortex motions. Ann. Geophys. 2011; 29(6):1029-1035, doi:10.5194/angeo-29-1029-2011. URL http://dx.doi.org/10.5194/angeo-29-1029-2011.

14. Mathioudakis M, Jess D, Erdélyi R. Alfvén waves in the solar atmosphere. Space Sci. Rev. 2013; 175(1-4):1-27, doi:10.1007/ s11214-012-9944-7. URL http://dx.doi.org/10.1007/s11214-012-9944-7.

15. Morton R, Verth G, Fedun V, Shelyag S, Erdelyi R. Evidence for the photospheric excitation of incompressible chromospheric waves. Astrophys. J. 2013; 768(1):17, doi:10.1088/0004-637X/768/1/17. URL http://dx.doi.org/10.1088/0004-637X/768/1/17.

16. Oreshina A, Oreshina I. Perspectives of current-layer diagnostics in solar flares. Astron. Astrophys. 2013; 558:16, doi:10.1051/ 0004-6361/201220348. URL http://dx.doi.org/10.1051/0004-6361/201220348.

17. Verth G, Erdèlyi R, Goossens M. Magnetoseismology: eigenmodes of torsional Alfvén waves in stratified solar waveguides. Astrophys. J. 2010; 714(2):1637-1648, doi:10.1088/0004-637X/714/2/1637. URL http://dx.doi.org/10.1088/0004-637X/714/2/1637.

18. Gamba A, Ambrosi D, Coniglio A, De Candia A, Di Talia S, Giraudo E, Serini G, Preziosi L, Bussolino F. Percolation, morphogenesis, and burgers dynamics in blood vessels formation. Phys. Rev. Lett. 2003; 90(11):118 101, doi:10.1103/PhysRevLett.90.118101. URL http://dx.doi.org/10.1103/PhysRevLett.90.118101.

19. Franchi F, Lazzari B, Nibbi R. Uniqueness and stability results for non-linear Johnson-Segalman viscoelasticity and related models. Discrete Contin. Dyn. Syst. Ser. B 2014; 19(7).

20. Koo J, Kleinstreuer C. Viscous dissipation effects in microtubes and microchannels. Int. J. Heat Mass Transfer 2004; 47(14):3159-3169, doi:10.1016/j.ijheatmasstransfer.2004.02.017. URL http://dx.doi.org/10.1016/j.ijheatmasstransfer.2004.02.017. 
21. Zheng $X, X i e ~ C . A$ viscoelastic model of blood capillary extension and regression: derivation, analysis, and simulation. J. Math. Biol. 2014; 68(1-2):57-80, doi:10.1007/s00285-012-0624-8. URL http://dx.doi.org/10.1007/s00285-012-0624-8.

22. Straughan B. Heat Waves, Applied Mathematical Sciences, vol. 177. Springer New York, 2011, doi:10.1007/978-1-4614-0493-4. URL http://dx.doi.org/10.1007/978-1-4614-0493-4.

23. Joseph DD, Preziosi L. Heat waves. Rev. Modern Phys. 1989; 61(1):41-73, doi:10.1103/RevModPhys.61.41. URL http://dx.doi. org/10.1103/RevModPhys.61.41.

24. Khayat R, Ostoja-Starzewski M. On the objective rate of heat and stress fluxes. Connection with micro/nano-scale heat convection. Discrete Contin. Dyn. Syst. 2011; 15:991-998., doi:10.3934/dcdsb.2011.15.991. URL http://dx.doi.org/10.3934/dcdsb.2011.15. 991.

25. Morro A. Evolution equations and thermodynamic restrictions for dissipative solids. Math. Comput. Model. 2010; 52:1869-1876, doi:10.1016/j.mcm.2010.07.021. URL http://dx.doi.org/10.1016/j.mcm.2010.07.021.

26. Christov C. On frame indifferent formulation of the Maxwell-Cattaneo model of finite-speed heat conduction. Mech. Res. Comm. 2009; 36(4):481-486, doi:10.1016/j.mechrescom.2008.11.003. URL http://dx.doi.org/10.1016/j.mechrescom. 2008.11.003.

27. Straughan B. Acoustic waves in a Cattaneo-Christov gas. Phys. Lett. A 2010; 374(26):2667-2669, doi:10.1016/j.physleta.2010.04.054. URL http://dx.doi.org/10.1016/j.physleta.2010.04.054.

28. Nield D, Kuznetsov A. An historical and topical note on convection in porous media. J. Heat Transfer 2013; 135:061 201 (10 pp), doi:10.1115/1.4023567. URL http://dx.doi.org/10.1115/1.4023567.

29. Fabrizio M, Lazzari B, Nibbi R. Thermodynamics of non-local materials: extra fluxes and internal powers. Contin. Mech. Thermodyn. 2011; 23(6):509-525, doi:10.1007/s00161-011-0193-x. URL http://dx.doi.org/10.1007/s00161-011-0193-x.

30. Coleman B, Owen D. A mathematical foundation for thermodynamics. Arch. Rational Mech. Anal. 1974; 54:1-104, doi:10.1007\% 2FBF00251256. URL http://dx.doi.org/10.1007\%2FBF00251256.

31. Fabrizio M, Franchi F, Straughan B. On a model for thermo-poroacoustic waves. Internat. J. Engrg. Sci. 2008; 46(8):790-798, doi:10.1016/j.ijengsci.2008.01.016. URL http://dx.doi.org/10.1016/j.ijengsci.2008.01.016.

32. Vázquez J. The porous medium equation: mathematical theory. Oxford University Press, 2007.

33. Hayat T, Afsar A, Ali N. Peristaltic transport of a Johnson-Segalman fluid in an asymmetric channel. Math. Comput. Modelling 2008; 47(3-4):380-400, doi:10.1016/j.mcm.2007.04.012. URL http://dx.doi.org/10.1016/j.mcm.2007.04.012.

34. Hina S, Hayat T, Asghar S. Peristaltic transport of Johnson-Segalman fluid in a curved channel with compliant walls. Nonlinear Anal. Model. Control 2012; 17(3):297-311.

35. Oreshina A, Somov B. On the heat conduction in a high-temperature plasma in solar flares. Astronomy Letters 2011; 37(10):726-736, doi:10.1134/S1063773711090064. URL http://dx.doi.org/10.1134/S1063773711090064.

36. Quintanilla R. Exponential stability in the dual-phase-lag heat conduction theory. J. Non-Equilib. Thermodyn 2002; 27:217-227, doi:10.1515/JNETDY.2002.012. URL http://dx.doi.org/10.1515/JNETDY.2002.012.

37. Fabrizio M, Franchi F. Delayed thermal models: Stability and thermodynamics. J. Thermal Stresses 2014; 37(2):160-173, doi: 10.1080/01495739.2013.839619. URL http://dx.doi.org/10.1080/01495739.2013.839619.

38. Tzou D. Macro-to-Microscale Heat Transfer: The Lagging Behavior. Taylor and Francis, Washington, 1997.

39. Nield D. Impracticality of MHD convection in a porous medium. Trans. Porous Media 2008; 73(3):379-380, doi:10.1007/ s11242-007-9181-9. URL http://dx.doi.org/10.1007/s11242-007-9181-9.

40. Nield D. A comment on "on magneto convection in a mushy layer" by d. n. riahi. Trans. Porous Media 2011; 89(2):287-288, doi:10.1007/s11242-011-9770-5. URL http://dx.doi.org/10.1007/s11242-011-9770-5. 